\title{
Hacia la construcción de indicadores habitacionales: principios y criterios desde el enfoque de derechos ${ }^{1}$
}

Towards the construction of housing indicators: principles and criteria from the rights approach

\section{María Laura Canestraro}

Grupo de Estudios Sociourbanos, Centro de Estudios Politicos y Sociales, Facultad de Humanidades (FH), Universidad Nacional de Mar del Plata (UNMdP)-CONICET,

Argentina

mlcanestraro@gmail.com

\section{Maria Laura Zulaica}

Grupo de Estudios Sociourbanos, Centro de Estudios Politicos y Sociales, Facultad de Humanidades (FH), Universidad Nacional de Mar del Plata (UNMdP) -Instituto del Hábitat y del Ambiente (IHAM), Facultad de Arquitectura, Urbanismo y Diseño (FAUD), Argentina

laurazulaica@yahoo.com.ar

\section{Maria Soledad Arenaza}

Grupo de Estudios Sociourbanos, Centro de Estudios Politicos y Sociales, Facultad de Humanidades (FH), Universidad Nacional de Mar del Plata (UNMdP), Argentina

sole_ar@hotmail.com

\section{ReSUMEN:}

La Nueva Agenda Urbana consensuada en Hábitat III destaca la necesidad de redefinir políticas públicas para las ciudades latinoamericanas. Uno de sus componentes centrales se vincula con la problemática del acceso al suelo. En este marco, el Índice de la Prosperidad Urbana cobra importancia al incorporar indicadores habitacionales tendientes a la sustentabilidad urbana. Sin embargo, prescinde del componente del suelo y de criterios cualitativos que, desde un enfoque de derechos, permitan complejizar el diagnóstico y la intervención urbana. Este artículo avanza sobre la propuesta de construcción de indicadores habitacionales definiendo principios y criterios útiles para monitorear el cumplimiento de políticas públicas.

Palabras ClaVe: Indicadores habitacionales, Suelo, Enfoque de derechos, Políticas públicas.

\section{ABSTRACT:}

The New Urban Agenda agreed upon at Habitat III highlights the need to redefine public policies for Latin American cities. One of the main topics of discussion is linked to the problem of access to land. In this framework, the Urban Prosperity Index gains importance by incorporating housing indicators aimed at urban sustainability. However, it does not include the land component and qualitative criteria that would allow, from a rights-based perspective, to make diagnosis and urban intervention more complex. This paper elaborates upon the proposal of construction of housing indicators by defining principles and useful criteria to monitor compliance with public policies.

KEYWORDS: Housing indicators, Land, Rights approach, Public policies. 


\section{INDICADORES: ¿QUÉ SON?, ¿PARA QUÉ SIRVEN?}

La importancia de contar con indicadores de desempeño cobra cada vez mayor centralidad a nivel global, dada la necesidad de medir el grado de avance en materia de compromisos y objetivos asumidos por los Estados en los diversos ámbitos que reúnen a la comunidad internacional. Algunas metas -como los 17 Objetivos de Desarrollo Sostenible (ODS) trazados por la Asamblea General de las Naciones Unidas para el período 2016-2030 - constituyen los desafíos más apremiantes de nuestro tiempo (Wood et al., 2018), de ahí la importancia de contar con indicadores que permitan medir los objetivos de progreso hacia el desarrollo sostenible y, en función de ello, priorizar las opciones de políticas públicas más efectivas y eficientes para lograr su cumplimiento (Reyers et al., 2017).

A la par de ello, el uso de indicadores ha cobrado protagonismo en el ámbito de los Estados Nacionales y de los gobiernos locales. En términos conceptuales, los indicadores pueden ayudar a entender y estructurar la definición de políticas públicas y la interpretación de tendencias para encontrar soluciones a los problemas, al mismo tiempo en que pueden brindar información relevante sobre los resultados alcanzados por la implementación de dichas políticas. En este marco, los indicadores de desempeño proveen información sobre áreas fundamentales de acción de los agentes públicos, tales como eficiencia, eficacia, calidad y economía de los recursos (Bonnefoy y Armijo, 2005).

Un uso novedoso de indicadores se presenta actualmente en el monitoreo del cumplimiento de sentencias judiciales que abordan problemáticas estructurales con un alcance colectivo, como ocurrió en Argentina con la sentencia en el caso "Mendoza" ${ }^{2}$. El fallo, dictado en 2008 por la Corte Suprema de Justicia de la Nación, condenó a distintas jurisdicciones y niveles del Estado a recomponer ambientalmente la cuenca MatanzaRiachuelo -y a mejorar la calidad de vida de sus habitantes- mediante la implementación de un plan de saneamiento ambiental que incluía disposiciones en ocho materias (información pública, saneamiento de basurales, limpieza de los márgenes del río, plan sanitario de emergencia, entre otras), y que fijaba plazos y prioridades a las intervenciones del Estado durante el proceso de implementación de la sentencia. En este marco, el máximo tribunal instó a la Autoridad de la Cuenca Matanza Riachuelo (ACUMAR), en su calidad de organismo encargado de implementar el plan de saneamiento de la cuenca, a adoptar alguno de los sistemas internacionales de medición disponibles, para poder evaluar el grado de cumplimiento de los objetivos ordenados en el fallo y luego informar al juzgado federal de ejecución de la sentencia (Merlinksy, 2013).

Por último, la información que proveen los indicadores es clave para el accionar de las organizaciones de la sociedad civil, como los organismos de derechos humanos, las organizaciones de mujeres, y diversas organizaciones especializadas en la promoción y protección de derechos, las cuales demandan metodologías para poder efectivizar un control ciudadano sobre las acciones de los Estados (Pautassi, 2013; 2016).

Desde el punto de vista operativo, un indicador es una variable que describe características del estado de un sistema a través de datos observados o estimados. Por su parte, un índice es una agregación cuantitativa de muchos indicadores que proporciona una visión simplificada, coherente y multidimensional de un sistema (Mayer, 2008).

En cuanto a la selección de los indicadores relevantes para el monitoreo de las políticas públicas, ésta puede obedecer a distintos enfoques y marcos conceptuales, como son los enfoques del ámbito del desarrollo y del de los derechos. En el primer caso, se buscará medir el desarrollo económico y en el segundo el cumplimiento de derechos en un Estado (Pautassi, 2016; Abramovich, 2006). Desde ya, la selección de los indicadores puede perseguir la convergencia de varios enfoques en el monitoreo de la política, lo que enriquecerá las retroalimentaciones necesarias para lograr los objetivos previstos (Shen et al., 2011). En este marco, el denominado "enfoque de derechos en las políticas y estrategias de desarrollo" considera al derecho internacional de los derechos humanos como un marco conceptual aceptado por la comunidad internacional, 
capaz de orientar el proceso de formulación, implementación y evaluación de las políticas en el campo del desarrollo (Abramovich, 2006).

\section{El Índice de Prosperidad Urbana}

Desde la primera edición celebrada en Vancouver en 1976, las conferencias Hábitat se realizan cada 20 años con el objetivo de promover el desarrollo de asentamientos humanos sostenibles en un contexto mundial de urbanización progresiva.

En 1996, en Hábitat II (Estambul) se acordaron siete compromisos y un plan de acción mundial, que estipulaban una serie de estrategias tendientes a lograrlos. Estos objetivos referían a: 1. Vivienda adecuada para todos; 2 . Asentamientos humanos sostenibles; 3. Habilitación de facultades y participación de actores clave públicos, privados y comunitarios; 4. Equidad de género; 5. Financiamiento de la vivienda y los asentamientos humanos; 6. Cooperación Internacional, y 7. Evaluación de los progresos. Con la misma perspectiva, en el año 2000 Naciones Unidas adoptó los ocho Objetivos de Desarrollo del Milenio para el período 2000-2015 (Karol, 2016).

Ya en el programa de Hábitat II se planteó la necesidad de garantizar el acceso a la tierra como una de las prioridades centrales, por considerarse que "todos los gobiernos deben mostrar su determinación de promover una oferta de terrenos adecuada en el contexto de las políticas de uso sostenible de la tierra”. Esto surge de la idea de que la falta de políticas de ordenamiento territorial en zonas rurales y urbanas es una de las principales causas de desigualdad y pobreza.

En 2015, la Asamblea General de Naciones Unidas aprobó 17 Objetivos de Desarrollo Sostenible (ODS) para el período 2016-2030, entre los que el número 11 apunta a lograr que las ciudades y los asentamientos humanos sean inclusivos, seguros, resilientes y sostenibles.

Con estos antecedentes, en la reunión de Hábitat III, realizada en Quito en 2016, se aprobó una nueva agenda urbana. Entre los componentes centrales de esta agenda, se destacan la Campaña Global Suelo Seguro impulsada por Hábitat para la Humanidad, y la ejecución de un plan de acción del Grupo Urbano de Instrumentos de Suelo de América Latina y el Caribe, perteneciente a la Red Global de Instrumentos de Suelo de ONU-Hábitat.

En la actualidad, los distintos países cuentan con metodologías propias de medición de la situación actual de las ciudades desde una perspectiva multidimensional. Esto permite identificar oportunidades y áreas potenciales de intervención para que las ciudades transiten por un sendero de prosperidad (ONU-Hábitat, 2016a), concepto que es definido como un estado de satisfacción socioeconómica unido a la realización de otras condiciones no materiales, que en conjunto brindan seguridad integral a las sociedades y a los individuos, en el presente, mediano y largo plazo (ONU-Hábitat, 2012).

En este contexto, una de las mayores preocupaciones radica en la posibilidad de medir adecuadamente diversos procesos urbanos que tienen como eje vertebrador al suelo. Con ese horizonte tomó impulso la utilización del Índice de la Prosperidad Urbana (CPI, City Prosperity Index, por sus siglas en inglés), creado en 2012 por iniciativa de Naciones Unidas para medir el progreso actual y futuro de las ciudades.

De acuerdo con las conceptualizaciones aportadas por el Programa ONU-Hábitat, una ciudad próspera es aquella que desarrolla en forma equilibrada un buen desempeño en estas seis dimensiones (ONU-Hábitat, 2016a; 2016b):

1. Una ciudad productiva que contribuye al crecimiento económico, genera ingresos, provee trabajos decentes e igualdad de oportunidades.

2. Una ciudad con infraestructura suficiente y eficiente para proporcionar los soportes físicos adecuados para la movilidad, la productividad, la interconectividad y los servicios básicos a la población. 
3. Una ciudad con buena calidad de vida que brinda a todos los ciudadanos una vivienda adecuada y servicios básicos decentes, facilitando el acceso igualitario a los servicios sociales, bienes públicos y buenas condiciones ambientales.

4. Una ciudad equitativa e incluyente para asegurar la distribución equitativa y la redistribución de los beneficios de una ciudad próspera y reducir la incidencia de la pobreza y de los asentamientos precarios.

5. Una ciudad ambientalmente sostenible que protege el ambiente urbano al tiempo que garantiza el crecimiento mediante el aprovechamiento sostenible de los recursos naturales.

6. Una ciudad con buena gobernanza que permite la participación ciudadana, administra los recursos en forma transparente, fortalece la capacidad institucional y hace más eficiente la gestión regulada.

En síntesis, la metodología de construcción de un índice de ciudades prósperas conlleva la medición de un conjunto de indicadores que abarcan las seis dimensiones hacia la prosperidad: 1) productividad; 2) infraestructura de desarrollo; 3) calidad de vida; 4) equidad e inclusión social; 5) sostenibilidad ambiental; y 6) gobernanza y legislación urbana. Cada una estas dimensiones incluye subdimensiones, y dentro de éstas se incorporan indicadores específicos medibles y comparables.

Utilizado actualmente en más de 300 ciudades del mundo, el CPI busca producir información y aportar soluciones a las formas ineficientes, insostenibles y disfuncionales con que fueron planificadas muchas ciudades del siglo pasado. Propone orientar cambios transformadores en ellas a través de un marco práctico para la formulación, implementación y seguimiento de un plan de acción que integre las políticas públicas y las acciones encaminadas a incrementar sus niveles de prosperidad, con base en un enfoque holístico e integrado, esencial para la promoción del bienestar colectivo y la satisfacción de todos (ONU-Hábitat, 2016a; 2016b).

$\mathrm{Al}$ igual que otros conceptos, la prosperidad urbana no deja de ser un concepto discutible e incluso cuestionable, al admitir cierta versatilidad, ambigüedad e idealización, lo que puede dificultar el análisis de situaciones concretas y el diseño de políticas urbanas. Estas críticas se hacen evidentes en la Nueva Agenda Urbana producto de la Conferencia Hábitat III, celebrada en 2016, en la que se prescinde de reconocer las ciudades actuales en sus condiciones marginalizadoras y segregadas, protectoras de intereses elitistas y desreguladoras, violentas e inseguras, exclusivas y excluyentes (Rodríguez y Sugranyes, 2017).

Dentro de los temas de enorme centralidad previamente incluidos y discutidos en la agenda internacional -que quedaron fuera del debate en Hábitat III- se encuentra del el suelo urbano. En rigor, la dinámica más regresiva que se ha dado a través de los 40 años transcurridos entre Hábitat I y Hábitat III fue el cambio en la importancia que se le asigna a la tierra como elemento esencial para el desarrollo y la sostenibilidad de los asentamientos humanos (Rodríguez y Sugranyes, 2017).

En este marco, en un trabajo previo (Canestraro et al., 2017) planteamos algunas reflexiones críticas en torno al Índice de Prosperidad Urbana, vinculadas tanto a la construcción del mismo como a la necesidad de incorporar otras dimensiones en la medición, a partir de la observación de su comportamiento en un área urbana de la ciudad de Mar del Plata (partido de General Pueyrredón, provincia de Buenos Aires, Argentina).

A continuación, nos proponemos avanzar sobre esas consideraciones preliminares realizando algunas observaciones e incorporando otras dimensiones relevantes para la construcción de indicadores habitacionales. Definimos principios y criterios para su diseño y utilización con base en la convergencia de enfoques.

\section{SOBRE EL CASO ANALIZADO}

La problemática del acceso al suelo urbano constituye uno de los ejes nodales del desarrollo urbano de la ciudad de Mar del Plata. Su fundación misma en 1874 se incluye dentro del fenómeno generalizado de los negocios de tierras a urbanizar (Irigoin, 1991), mientras que la propia dinámica de su urbanización 
se vinculó estrechamente con la producción y concentración de suelo urbano (Fernández et al., 1996; Mariano, 2000; Núñez, 2000). Desde entonces, y hasta avanzadas las primeras décadas del siglo XX, hubo una importante incidencia de propietarios en la subdivisión y venta de tierras y en la generación de nuevos polos de urbanización (Mariano, 2000), que se anticiparon, incluso, a los procesos de asentamiento de la población (Núñez, 2000) ${ }^{3}$.

Entre las décadas de 1930 y 1960 los sectores populares pudieron acceder al suelo a través de la subasta, el alquiler de la tierra, y la compra de lotes en mensualidades ubicados en zonas sin infraestructura de servicios ni aptas para la residencia (Núñez, 2000). La configuración de la zona elegida, denominada Distrito 5.1. Oeste Interno ${ }^{4}$, se inscribe en la progresiva periurbanización de la ciudad que se originó a partir de la década de 1950, y que transformó el uso de suelo rural a urbano más bien como parte de un proceso de especulación, que como consecuencia de la expansión de la planta urbana (Núñez, 1994). Aunque muchos hogares resolvieron su demanda de vivienda a través de la compra de lotes en mensualidades en la periferia, y aunque esto produjo un aumento relativo de la cantidad de propietarios en el partido en detrimento de los inquilinos, ese incremento se dio en zonas sin infraestructuras ni aptitudes para la localización residencial. En consecuencia, no trajo aparejada una mejora habitacional (Núñez, 2000).

A partir de la década de 1970 se inició un proceso de redistribución de la población que propició la expansión de la ciudad y la emergencia de pequeñas localidades contiguas. En ese contexto, se produjeron diversas formas de hábitat popular, en su mayoría bajo la modalidad de autoconstrucción y proyectos habitacionales de vivienda social subsidiados por el Estado (Fernández et al., 1996). De manera progresiva, las principales formas de acceso al suelo -compra de lotes en mensualidades, acceso a vivienda de interés social y ocupación de tierras- supusieron procesos de menor integración a la ciudad, que confluyeron en una expansión de la periferia como "zona reservada" a los sectores de bajos recursos, con servicios e infraestructura inexistentes o deficientes.

Aunque la periferia se ha ido urbanizando progresivamente en el transcurso de las décadas, aún persiste un fuerte déficit urbano habitacional que evidencia la desigualdad histórica en el acceso a una vivienda adecuada bajo el modelo de urbanización del partido de General Pueyrredón.

El suelo constituye un elemento central en los procesos de urbanización, por lo que resulta esencial abordarlo, no sólo como soporte del asentamiento y la aglomeración social (Pírez y Salazar, 2016) sino, además, en función de la incidencia que tiene la propiedad del suelo en la "selectividad" de las intervenciones estatales. El caso analizado indaga en esta segunda observación, y sugiere como hipótesis que las condiciones habitacionales de una vivienda mejoran con la propiedad del suelo, debido a las estrategias familiares y/o a la acción del Estado.

Aunque los datos existentes, extraídos del censo, impiden confirmar esta hipótesis -en la medida en que no relacionan la propiedad del suelo y la vivienda con el acceso a servicios e infraestructuras-, la condición de propietario es un requisito excluyente para el acceso a determinados servicios domiciliarios como, por ejemplo, la red pública de gas. De ahí la selección y utilización de este indicador en la metodología aplicada al caso analizado.

Desde ya que la estrategia metodológica de este trabajo no supone el desconocimiento de otras formas de acceso al suelo urbanizado y a la vivienda distintas de la propiedad, sin embargo, y a pesar de esta limitación, la propiedad del suelo es un indicador relevante y eficaz a los efectos de evidenciar la centralidad del componente suelo en la infraestructura de la vivienda como subdimensión de la "prosperidad urbana", tal como se constatará en el caso analizado. 


\section{Observación 1. La omisión del suelo en el análisis de la infraestructura de vivienda}

En línea con las metodologías de medición de la situación actual de las ciudades desde una perspectiva multidimensional se encuentran los índices sintéticos o indicadores compuestos, que contienen información agregada sobre determinados fenómenos y procesos sociales.

Un indicador compuesto es una representación simplificada que busca resumir un concepto multidimensional en un índice simple (unidimensional) con base en un modelo conceptual subyacente (Schuschny y Soto, 2009). En función de ello, los índices permiten monitorear los progresos realizados a partir de metas definidas previamente, con lo que se convierten en instrumentos indispensables para la toma de decisiones (Spangenberg y Bonniot, 1998). Además, permiten definir umbrales (Srebotnjak et al., 2010), es decir, puntos de inflexión relevantes en la implementación de políticas establecidas (Donatiello, 2004) que adquieren un valor comparativo.

En su aplicación al caso analizado, en primer lugar se estudió la metodología de ONU-Hábitat (2012) en la Iniciativa de Ciudades Prósperas, aplicada a diferentes casos a nivel mundial, pero con fuerte presencia en México (ONU-Hábitat, 2016a) y Ecuador (ONU-Hábitat, 2016b). Luego, se identificaron las dimensiones, subdimensiones e indicadores aportados por la misma.

Tal como ha sido señalado en el apartado previo, el Índice de la Prosperidad integra este concepto a partir de la evaluación de seis dimensiones, una de las cuales es la Infraestructura de Desarrollo. A su vez, cada dimensión incluye subdimensiones. Entre ellas la infraestructura de vivienda resulta una de las subdimensiones de la infraestructura de desarrollo, en la cual se engloban cuatro indicadores: vivienda durable, acceso a agua mejorada, espacio habitable suficiente y densidad poblacional.

La primera observación a realizar sobre esta subdimensión es que el subíndice referido a la infraestructura de vivienda no incorpora el componente suelo como variable central en el abordaje de la prosperidad. En una marcada contradicción con la relevancia dada al suelo en los ODS y la agenda de ONU-Hábitat, este componente ha sido omitido en el análisis de los instrumentos de medición propuestos por las mismas agencias y organismos que impulsan dichas agendas, tal como profundizamos en un trabajo reciente (Canestraro et al., 2017).

Vale aclarar que, si bien el problema del suelo atraviesa transversalmente a las seis dimensiones que hacen a la "prosperidad urbana" -en tanto constituye un eje nodal del acceso y la integración a la ciudad (DurandLasserve, 1997)-, lo incorporamos al subíndice referido en función de los datos existentes, priorizando la factibilidad de lograr un correlato empírico de aquello que pretendemos problematizar, tal como lo señaláramos al presentar el caso analizado.

Metodológicamente, Dizdaroglu (2015) destaca que un marco de indicadores válidos debe cumplir con los siguientes criterios básicos: 1) relevancia y utilidad; 2) solidez analítica, y 3) mensurabilidad. Si tenemos esto en consideración al analizar la validez del subíndice referido a la infraestructura de vivienda, y tomamos los últimos datos oficiales existentes en nuestro país (INDEC, 2010), se perciben controversias al contrastarlos con la variable relativa al régimen de tenencia del suelo y la vivienda, el cual manifiesta una distribución distinta. Es decir, el análisis de la situación del área de estudio respecto de la subdimensión infraestructura de la vivienda muestra diferencias cuando se incorpora una variable de acceso al suelo.

En primer lugar, los indicadores correspondientes a la subdimensión infraestructura de vivienda se ajustaron en función de la información disponible y factible de procesar a nivel de radios censales (INDEC, 2010). En este procedimiento se partió de un estudio previo realizado en el área (Canestraro et al., 2017). En ese estudio los indicadores considerados para la construcción del índice son: calidad constructiva insuficiente, acceso a agua de red pública, hacinamiento severo y densidad de población.

Una vez procesada la base de datos, se analizó el comportamiento espacial de los indicadores mencionados mediante el uso de un sistema de información geográfica (gvSIG versión 1.11). A fin de poder 
establecer comparaciones, los valores de los indicadores se estandarizaron para transformarlos en unidades adimensionales, mediante la técnica de puntaje omega (Buzai, 2003).

Una vez calculados los valores estandarizados de los indicadores se elaboró un índice sintético conformado por el promedio de los mismos. Posteriormente, se incorporó al análisis el indicador "porcentaje de hogares con propiedad de la vivienda y del terreno" como un aspecto relevante en términos del acceso a las infraestructuras de la vivienda, a fin de observar la incidencia del componente suelo, que es omitido por el Índice de Prosperidad Urbana. En función de ello, analizamos qué sucedía al incorporarse este indicador en los datos disponibles para un sector del ejido urbano de la ciudad de Mar del Plata-Distrito 5.1. Oeste Interno-. A partir del relevamiento de esa condición, esta incorporación nos permite realizar inferencias respecto de una incidencia más generalizada ${ }^{5}$ (Figura 1 ).

Cabe aclarar que en este trabajo tomamos una de las varias categorías del régimen de tenencia disponibles en el censo, y excluimos al propietario solo de la vivienda, al inquilino, al ocupante por préstamo o cesión, al ocupante por trabajo o relación de dependencia, y otra situación. Asimismo, se toma en cuenta que en el caso de la legislación argentina, la seguridad jurídica de la tenencia se encuentra protegida en diferentes grados para quienes detentan la condición de propietario, poseedor (ocupante) e inquilino.

\section{FIGURA 1}

\section{Distrito 5.1 Oeste Interno}

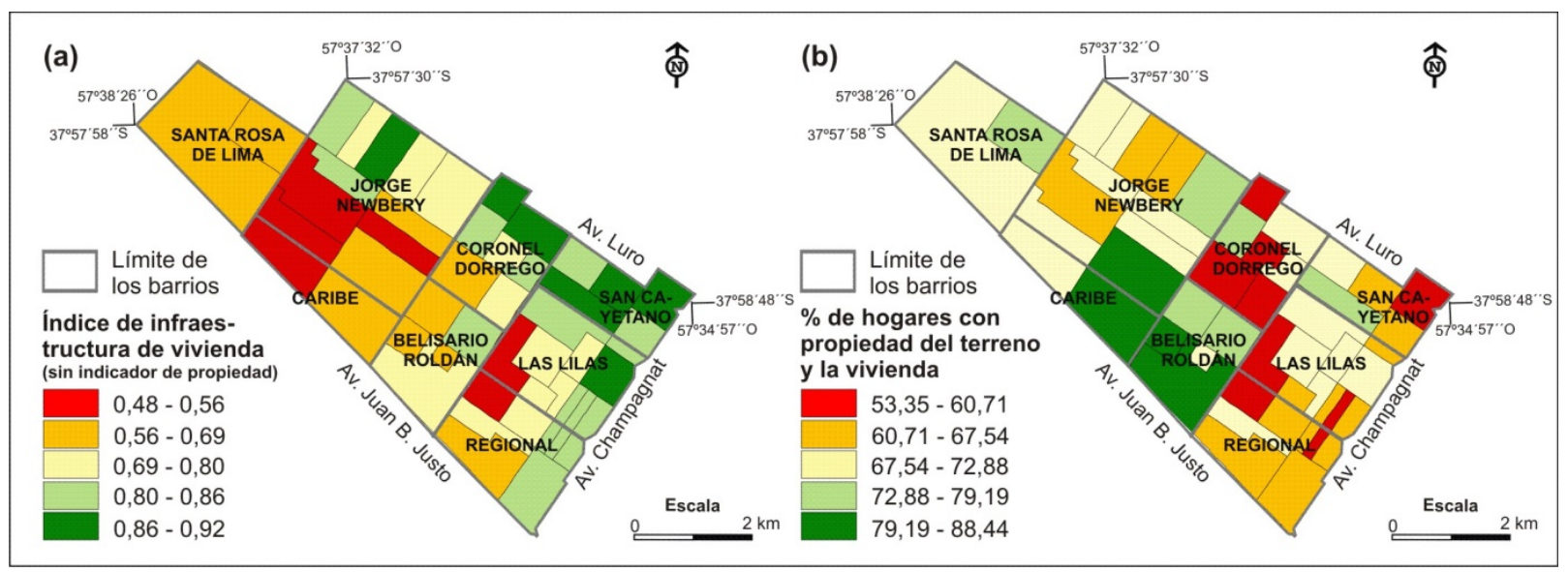

Índice construido a partir de la integración de los indicadores de la subdimensión infraestructura de vivienda (a) e indicador referido a la propiedad del terreno y la vivienda (b)

Canestraro et al. (2017) 
Al incorporar el indicador propiedad de la vivienda y del terreno al índice estandarizado de infraestructura de vivienda se verifica en situación desfavorable un sector importante de la zona (ver Figura 1). Asimismo, las diferencias en el cálculo del índice considerado - sin tener en cuenta esta variable e incorporándolamuestra las diferencias en su distribución. Mientras que la mayor parte revela mejoras en la subdimensión infraestructura de vivienda, existen extensos sectores en los que las situaciones críticas se profundizan (Figura 2). De lo anterior se desprende que el comportamiento de los indicadores exhibe resultados distintos si se introduce la propiedad de la vivienda y del terreno a la subdimensión de la infraestructura de vivienda ${ }^{6}$.

En el marco de un enfoque holístico e integrado como el que supone la metodología del CPI, el acceso al suelo debería ser considerado como un factor decisivo en la integración a la ciudad (Durand-Lasserve, 1997), a partir del cual se articulan otros derechos ciudadanos. No obstante esto, es aquí donde las controversias se hacen evidentes, ya que la metodología citada, así como otras aplicadas a las ciudades latinoamericanas -como la Iniciativa de Ciudades Emergentes y Sostenibles (BID, 2014)-, no incorpora el acceso al suelo urbano como un indicador relevante de la prosperidad ni de la sustentabilidad urbana.

FIGURA 2

Distrito 5.1 Oeste Interno

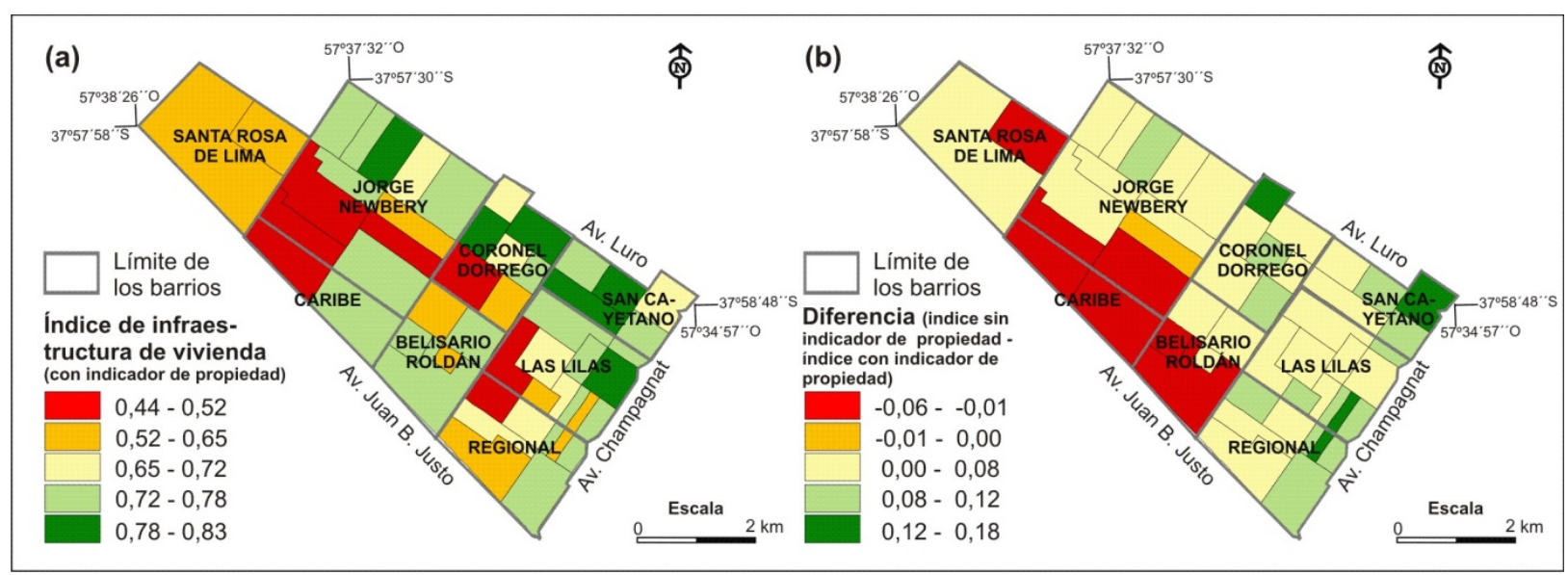

Índice de infraestructura de vivienda incorporando el indicador referido a propiedad (a) y diferencia en los índices calculados (b) Canestraro et al. (2017)

Los escasos estudios existentes sobre indicadores de suelo refieren mayoritariamente a sus usos en el ámbito rural. Respecto del suelo urbano, existen indicadores relativos al funcionamiento y monitoreo del mercado (Trivelli, 2007), pero no relevan su incidencia en términos del acceso e integración a la ciudad. Esta dimensión además debería tomar en cuenta aquellas prácticas que quedan por fuera de la lógica mercantil, ya sean predominantemente estatales o por necesidad $\left(\right.$ Abramo, 2003) ${ }^{7}$, o bien combinadas o superpuestas ${ }^{8}$. 


\section{Observación 2. Limitaciones en la selección y construcción de los indicadores que conforman el CPI}

Es importante resaltar algunas limitaciones que se encuentran implícitas en la selección y construcción de indicadores -en este caso habitacionales-, que aporten al análisis de la prosperidad urbana. Entre ellas se pueden mencionar aquellas que se vinculan con la indefinición del objetivo a medir, con las unidades de análisis y escalas adoptadas, con la disponibilidad de información sistematizada, con los vínculos institucionales y enfoques adoptados en la construcción. A continuación, se describen brevemente cada una de estas limitaciones.

\section{Objetivo a medir}

La indefinición operativa de la prosperidad urbana conlleva múltiples interpretaciones que necesariamente demandan ajustes en la selección de indicadores, ya que no existen criterios universales. Si bien las dimensiones que engloba el concepto de prosperidad urbana contribuyen a dar una respuesta a esta limitación, no es menos cierto que los objetivos de prosperidad pueden variar según los distintos contextos sociales y escalas territoriales.

\section{Unidades de análisis y escalas}

En relación a la escala territorial, es importante señalar que la elección de las unidades de análisis puede estar sesgada a los objetivos de quien las selecciona o construye, lo que incidiría en los resultados finales. En el estudio de caso que dio basamento a este trabajo (Canestraro et al., 2017) se utilizaron para la subdimensión infraestructura de vivienda los radios censales que son las unidades mínimas de las que se puede obtener información significativa, pero no siempre los indicadores son fácilmente adaptables a esa escala territorial, y en ocasiones no alcanzan a capturar las diferencias que existen al interior del espacio analizado.

Otro tema importante que surge del uso de indicadores es el enfoque multiescalar. Generalmente los indicadores suelen diseñarse en una escala determinada, y ello hace que su aplicación no tenga la suficiente correspondencia con la escala seleccionada para el conjunto de indicadores. La falta de perspectiva multiescalar en la definición de indicadores puede dar lugar a inexactitudes, lo que incidiría en la formulación de políticas y acciones concretas (Mori y Christodoulou, 2012). El CPI está pensado a una escala urbana y presenta limitaciones en sus aplicaciones en la escala intraurbana, en especial porque no capta las diferenciaciones en el interior de la ciudad. En este sentido, los valores promedio no siempre representan la realidad del conjunto y pueden subestimar situaciones críticas.

\section{Acceso a la información}

Otro problema común a la definición de indicadores es la ausencia o carencia de datos. No siempre es posible la recopilación de datos comparables temporal y espacialmente. Estos obstáculos se presentan, por ejemplo, cuando existen cambios en la metodología utilizada para la medición de datos a lo largo del tiempo. Además, en general, los datos se encuentran dispersos y esto dificulta el proceso de análisis y de síntesis. 


\section{$I V$. Coordinación institucional}

Como otra limitación significativa se plantea la dificultad en la coordinación entre instituciones (King, 2016), para generar y utilizar datos compatibles sumando esfuerzos en su recolección y análisis. Al respecto, no debe perderse de vista el hecho de que las políticas urbanas han sido diseñadas e implementadas tradicionalmente por una multiplicidad de organismos estatales que intervienen en el desarrollo de los procesos de urbanización de manera sectorial, de ahí que existe una alta dispersión de datos en este campo, agravada por el deslinde y la superposición de competencias jurisdiccionales -características de la organización de un sistema federal-, como ocurre en el caso de Argentina.

\section{$V$. Complementariedad de enfoques}

Por último, a todo lo anterior se suman las dificultades para lograr la validación de los indicadores desde un enfoque participativo, lo cual limita las posibilidades reales de implementación en políticas concretas. Esto no es una cuestión menor, ya que comprender la utilidad real y potencial de los sistemas de indicadores dentro de procesos de gobernanza multiactorales permite consensuar y construir respuestas conjuntas (Holden, 2013) para encontrar soluciones a problemas que amenazan la prosperidad de las ciudades. En consecuencia, es importante sumar estrategias participativas además del enfoque técnico-científico, a fin de incorporar las aspiraciones de los actores en un contexto determinado.

\section{Observación 3. La ausencia de un enfoque de derechos en el CPI}

Las agencias de Naciones Unidas y otras agencias de cooperación internacional han remarcado en los últimos años la importancia de promover un dialogo creciente entre los enfoques de desarrollo y de derechos humanos.

La diferencia que se plantea entre los enfoques de desarrollo y de derechos, gira en torno al marco conceptual que rodea a las políticas y estrategias que cada uno adopta para intervenir sobre problemas, la evaluación diagnóstica que hacen de los mismos, los objetivos que persiguen con dichas políticas, y la evaluación de desempeño en la que basan el éxito o fracaso de las intervenciones del Estado. Mientras lo habitual en las políticas y estrategias de desarrollo es que éstas apunten a lograr mejoras en los indicadores socioeconómicos relevantes, las políticas con un enfoque de derechos buscarán, precisamente, garantizar la efectividad de derechos (Pautassi, 2016; Abramovich, 2006).

En este marco, la metodología utilizada por el CPI se anuncia como un índice basado en los principios fundamentales de los derechos humanos, y en el contenido específico de derechos codificados en los tratados internacionales de derechos humanos. En función de ello, en lo que sigue de este apartado analizaremos si efectivamente se ha incorporado un enfoque de derechos humanos al CPI.

\section{A. La prosperidad urbana y el derecho a una vivienda adecuada}

La búsqueda de un diálogo creciente entre los enfoques de desarrollo y derechos humanos nos permite trazar un paralelismo entre el concepto de prosperidad acuñado por ONU-Hábitat -y utilizado por el CPI-, propio del campo del desarrollo, y el derecho a un "nivel de vida adecuado" proveniente del campo de los derechos humanos, y aplicado especialmente a los derechos económicos, sociales y culturales.

Como ha sido desarrollado previamente, el concepto de prosperidad refiere a un estado de satisfacción socioeconómica unido a la realización de otras condiciones no materiales, que en conjunto brindan seguridad 
integral a las sociedades y a los individuos en el presente, mediano y largo plazo (ONU-Hábitat, 2012). Por su parte, el "derecho a un nivel de vida adecuado" consagrado en dos de los principales instrumentos internacionales de derechos humanos - como la Declaración Universal de los Derechos Humanos (art. 25.1) y el Pacto Internacional de Derechos Económicos, Sociales y Culturales (PIDESC, art. 11, primer párrafo)engloba el acceso a la alimentación, vestido y vivienda adecuados, y a una mejora continua de las condiciones de existencia ${ }^{9}$.

Al igual que la prosperidad, el derecho a un nivel de vida adecuado también es un concepto multidimensional. Sin embargo, y a diferencia de aquél, se define en base al goce de un conjunto de derechos considerados indivisibles a fin de asegurar a cada persona y a su familia el bienestar o la ausencia de miseria.

Dentro del abanico de derechos comprendidos en el derecho a un nivel de vida adecuado, el derecho a una vivienda es, sin dudas, el que mejor dialoga con la integralidad del enfoque adoptado en la Iniciativa por la Prosperidad Urbana, en lo que refiere al menos a las problemáticas urbanas que ésta busca transformar.

De acuerdo al Comité de Derechos Económicos, Sociales y Culturales (Comité DESC) -el cual se constituye como principal órgano de interpretación y garantía del PIDESC-, el concepto de vivienda

...adecuada no se debe interpretar en un sentido estricto o restrictivo que lo equipare, por ejemplo, con el cobijo que resulta del mero hecho de tener un tejado por encima de la cabeza o lo considere exclusivamente como una comodidad. Debe considerarse más bien como el derecho a vivir en seguridad, paz y dignidad en alguna parte (...) significa disponer de un lugar donde poderse aislar si se desea, espacio adecuado, seguridad adecuada, iluminación y ventilación adecuadas, una infraestructura básica adecuada y una situación adecuada en relación con el trabajo y los servicios básicos, todo ello a un costo razonable (Comité DESC, 1991, párr. 7).

Asimismo, corresponde tener presente que el Comité DESC ha desarrollado los principales componentes que se encuentran incluidos en el concepto de "adecuación" de una vivienda a los efectos del PIDESC, en la Observación General Nro. 4 sobre el derecho a una vivienda adecuada. Estos aspectos abarcan a: a) la seguridad jurídica de la tenencia; b) la disponibilidad de servicios, materiales, facilidades e infraestructura; c) los gastos soportables; d) la habitabilidad; e) la asequibilidad; f) el lugar, y g) la adecuación cultural (Comité DESC, 1991, párr. 8).

En síntesis, si la Iniciativa por la Prosperidad Urbana busca intervenir y promover cambios en ciudades con escasez de infraestructura, equipamientos y viviendas adecuadas, contaminación ambiental, etc., el marco conceptual del derecho a una vivienda adecuada busca promover la realización de este derecho, estableciendo obligaciones a cargo de los Estados que involucran la adopción de políticas públicas respetuosas de su contenido.

Como consecuencia de ello, resulta lógico presuponer que el CPI -en tanto marco metodológico y conceptual para la producción de información relevante que busca orientar políticas y estrategias de desarrollo urbano con un enfoque de derechos-incluya los principios fundamentales de derechos humanos como ejes transversales de análisis, y, a su vez, que aquellas dimensiones específicas de esta metodología -vinculadas al desarrollo de infraestructura, calidad de vida, equidad e inclusión social y sostenibilidad ambiental-incorporen a su análisis el contenido de los componentes o atributos relevantes del derecho a una vivienda adecuada.

\section{B. Los Principios Generales de Derechos Humanos como ejes transversales de análisis}

Los tratados internacionales de derechos humanos consagran diversos principios medulares como la igualdad y no discriminación, la participación, y el acceso a mecanismos de exigibilidad, que los tomadores de decisiones deben observar de manera transversal en la elaboración e implementación de políticas públicas con un enfoque de derechos. 
En el marco de una convergencia de enfoques, la contribución de estos principios al campo de las políticas de desarrollo tiene dos potencialidades. Por un lado, estos principios constituyen elementos valiosos para pensar en los diversos componentes de una estrategia de desarrollo, y, por el otro, contribuyen a definir las obligaciones que tienen los Estados frente a los principales derechos humanos involucrados en una política de desarrollo (Abramovich, 2006).

\section{El principio de igualdad y no discriminación}

El principio de inclusión que suele regir a las políticas de desarrollo puede resultar enriquecido al vinculárselo con el principio de igualdad y no discriminación, consagrado en prácticamente todos los tratados internacionales de derechos humanos ${ }^{10}$. De acuerdo con este principio, los derechos deben ser reconocidos a todo individuo por el solo hecho de ser persona, sin discriminación alguna por motivos de raza, color, sexo, idioma, opinión política o de otra índole, origen nacional o social, posición económica, nacimiento, discapacidad física o mental, estado de salud (incluido el VIH/SIDA), orientación sexual, estado civil o cualquier otra condición política, social o de otro tipo. La igualdad y no discriminación no sólo imponen a los Estados la obligación de no discriminar (no implementar políticas y medidas discriminatorias o que tengan efectos discriminatorios), sino también la de proteger a las personas frente a prácticas o conductas discriminatorias por parte de terceras personas, sean agentes públicos o actores no estatales (Vázquez y Delaplace, 2011). Por último, el principio de igualdad y no discriminación obliga a los Estados a adoptar medidas concretas de protección en relación a los grupos que requieren atención prioritaria o especial en el ejercicio de los derechos en un determinado momento histórico (IPPDH, 2014).

Cada una de estas obligaciones aporta parámetros útiles para definir y evaluar políticas urbanas sostenibles. En el ámbito del derecho a la vivienda, las políticas deberían garantizar cierto grado de prioridad al acceso a una vivienda para los grupos desfavorecidos (Comité DESC, 1991, párr. 8). En relación a las infraestructuras y servicios, las políticas de abastecimiento de agua y saneamiento deberían priorizar a quienes no tengan acceso a agua potable (ACNUDH, 2011). Del mismo modo, la incorporación de este principio a una política de movilidad urbana obliga a considerar la disparidad existente en la extensión de redes de transporte público, y la calidad del servicio prestado entre diferentes regiones geográficas o sectores postergados de una misma ciudad (Hernández, 2017). En todos estos casos, el principio de igualdad tiene derivaciones concretas en cuanto a los criterios con los que deben distribuirse los presupuestos y el gasto social.

Otra de sus derivaciones en el campo de las políticas urbanas obliga a los Estados a proteger a las personas frente a prácticas o conductas discriminatorias por parte de terceras personas, sean agentes públicos o actores no estatales. La violencia de género que se presenta en el transporte público en diferentes ciudades demanda la adopción de medidas específicas que protejan a las mujeres en este ámbito (Rozas Balbontín el al., 2015). Otro ejemplo claro remite a la ordenación de los recursos hídricos en contextos de escasez de agua potable. En estos contextos, el Estado debe intervenir a fin de que se otorgue prioridad a los usos personales y domésticos del agua entre los distintos usos que compiten entre sí (ACNUDH, 2011).

\section{El principio de participación}

El derecho de participación y consulta en los asuntos públicos está consagrado en diversos instrumentos internacionales de derechos humanos ${ }^{11}$. Su incorporación como principio a las políticas públicas es una consecuencia política y jurídica del empoderamiento que otorga la adopción de un enfoque de derechos, al considerar a las personas como sujetos de derechos y no como beneficiarias de concesiones que hacen los Estados. 
En el ámbito de las políticas urbanas cobra cada vez mayor relevancia el principio de "gestión democrática de la ciudad", entendido como principio y fundamento estratégico del derecho a la ciudad, consagrado en la Carta Mundial por el Derecho a la Ciudad, adoptada en 2004 por las organizaciones y movimientos urbanos nucleados en el Foro Social Mundial.

Las estrategias y políticas de desarrollo pueden valerse de este principio como un método para identificar necesidades y prioridades a nivel local o comunitario (Abramovich, 2006), lo que resulta crucial para establecer qué asuntos se incorporan en las agendas gubernamentales, y, a su vez, para incidir en la definición misma de los problemas (IPPDH, 2014). Los presupuestos participativos son un buen ejemplo de la materialización de una gestión democrática de la ciudad en la instancia de definición de los problemas y objetos de intervención de las políticas urbanas.

Asimismo, las personas afectadas en sus derechos pueden aportar conocimiento significativo sobre los problemas que las afectan, y las alternativas de solución, al definirse la modalidad de abordaje que tendrá una política sobre un problema determinado (IPPDH, 2014). Un ejemplo claro de esto, es la participación de las personas afectadas por desalojos forzosos en la definición de los procesos de relocalización cuando el Estado garantiza una solución habitacional alternativa. En rigor, a lo largo de la implementación de una política, el principio de participación debe verse reflejado necesariamente en la apertura de espacios y canales que permitan tomar en cuenta las opiniones, percepciones y necesidades de la población destinataria y de la comunidad en su conjunto (IPPDH, 2014). En contextos de privatización de los servicios de suministro de agua, por ejemplo, los Estados deben impedir abusos que menoscaben el acceso al agua potable mediante un sistema regulador eficaz que prevea una supervisión independiente y una auténtica participación pública (Comité DESC, 2002).

Por último, la participación en los distintos formatos existentes de evaluación de las políticas se constituye como una instancia de rendición de cuentas hacia la ciudadanía, lo que permite reformular estrategias -a partir del grado de cumplimiento de los objetivos propuestos y en la experiencia acumulada durante el proceso de implementación de la política-, y dotar de una mayor eficiencia los recursos destinados a la misma.

Desde ya que la participación en todas las instancias aludidas presupone el ejercicio de otros derechos, tales como el derecho de asociación, de reunión, la libertad de expresión y el derecho de acceso a la información, entre otros. Por lo tanto, se torna central para este principio la producción de información por parte del Estado, su publicidad, y accesibilidad por parte de la ciudadanía (Pautassi, 2016; Abramovich, 2006).

\section{El principio de rendición de cuentas}

Una de las principales cuestiones para explorar la potencialidad de la relación entre derechos y políticas de desarrollo está en los mecanismos de rendición de cuentas y de responsabilidad (Abramovich, 2006).

La consecuencia lógica de considerar que las personas son titulares de derechos las empodera para demandar determinadas conductas y prestaciones. Como contracara de esto, las acciones que emprenda el Estado tienen un correlato en obligaciones jurídicas, imperativas y exigibles impuestas por los tratados de derechos humanos. A los efectos de garantizar su exigibilidad por parte de la ciudadanía, el Estado tiene la obligación de organizar el aparato institucional - para que las personas puedan recurrir a dispositivos administrativos o judiciales a fin de hacer valer sus derechos- removiendo los obstáculos sociales o económicos que dificultan la posibilidad de acceso a la justicia en un sentido amplio. 


\section{$I V$. La incorporación de los principios de ddhb en el CPI}

Al contrastar el CPI con un enfoque de derechos, la primera observación que surge es la falta de incorporación de estos principios como ejes transversales a las dimensiones que analiza, y respecto de las cuales produce información.

El hecho de que una de sus dimensiones indague en la equidad y la inclusión social - a partir de indicadores como la tasa de pobreza, viviendas en tugurios, el desempleo juvenil, entre otros- no es equiparable a lo que permitiría un enfoque transversal sobre la igualdad y la no discriminación aplicado a cada una de las dimensiones que son indagadas por el CPI.

Si tenemos en cuenta esta diferencia, puede suceder que el resultado de cualquiera otra de las dimensiones, como la de infraestructura del desarrollo, sea relativamente sólido, y, sin embargo, que ese resultado invisibilice patrones de exclusión de ciertos grupos al acceso a agua potable o a condiciones de vivienda durables, indicadores ambos que integran la subdimensión de infraestructura en vivienda.

El principio de participación y rendición de cuentas o acceso a la justicia tampoco constituye un eje transversal de análisis en el CPI. En rigor, ni siquiera se ve reflejado en los indicadores utilizados en la dimensión que analiza la gobernanza de las ciudades y la legislación urbana; solamente la participación electoral se toma en cuenta como indicador para medir la participación y rendición de cuentas.

Por último, y a modo de observación general, corresponde poner de relieve el hecho de que los informes que dan cuenta de los resultados obtenidos por la aplicación del CPI en las diferentes ciudades y países no utilizan un lenguaje con enfoque de derechos.

\section{La seguridad juridica de la tenencia como condicionamiento de la infraestructura de la vivienda}

$\mathrm{Al}$ incorporar la propiedad del terreno y la vivienda a la medición de la infraestructura de vivienda, hemos evidenciado cómo la medición de este indicador varía sensiblemente. Sin perjuicio de esto, si inscribimos la cuestión en un enfoque más amplio de la problemática habitacional, podemos matizar la centralidad de la propiedad del suelo en relación a la incidencia que ella tiene en los procesos de urbanización. Existen abundantes experiencias que apoyan el argumento de que la regularización de la propiedad no es una condición necesaria para la consolidación de los asentamientos o el mejoramiento de las viviendas (Arqueros y Canestraro, 2017). En tal sentido, se destaca que la introducción de servicios públicos y la realización de obras de infraestructura y mejoramiento tienen similar eficacia que la regularización, ya que otorgan legitimidad al asentamiento por parte del gobierno como un hecho consumado (Di Virgilio et al., 2015; Gilbert, 2002; Duhau, 2002 citados en Arqueros y Canestraro, 2017). También en este marco se ubica el "debate sobre los títulos", que ha desmitificado la relación entre el acceso a la propiedad legal de los terrenos y la asignación de recursos económicos a las familias para realizar mejoras en sus viviendas (Ostuni y Van Gelder, 2009; De Souza, 2001; Payne, 2001 citados en Arqueros y Canestraro, 2017).

Sin embargo, sí es necesario remarcar la centralidad que adquiere la seguridad en la tenencia de la tierra y de la vivienda en los procesos colectivos de urbanización, y, por consiguiente, en el acceso a las infraestructuras de la vivienda, sobre todo en aquellas regiones de mundo que presentan altos niveles de informalidad. Tal como ha sido señalado, el marco conceptual de los derechos humanos puede contribuir no sólo aportando principios generales, sino también definiciones respecto del contenido específico de los derechos que deben ser medidos. Ya en su Observación General Nro. 4, el Comité DESC señaló que todas las personas deberían gozar de cierto grado de seguridad de tenencia que les garantizara una protección legal contra el desalojo forzoso, el hostigamiento u otras amenazas (Comité DESC, 1991, párr. 8). 
Posteriormente, en la Observación General $N^{\circ} 7$, dedicada exclusivamente a desarrollar el contenido específico de las obligaciones de los Estados derivadas de la seguridad jurídica de la tenencia, el Comité DESC definió a los "desalojos forzosos" como el hecho de hacer salir a personas, familias y/o comunidades de los hogares y/o las tierras que ocupan, en forma permanente o provisional, sin ofrecerles medios apropiados de protección legal o de otra índole, ni permitirles su acceso a ellos (Comité DESC, 1997: párr. 3). Como componente del derecho a una vivienda adecuada, la seguridad jurídica de la tenencia obliga a los Estados a abstenerse de llevar a cabo desalojos forzosos, garantizar que se aplique la ley a sus agentes o a terceros que efectúen desalojos forzosos (Comité DESC, 1997: párr. 8), y a adoptar medidas legislativas que brinden la máxima seguridad de tenencia posible a los ocupantes de tierras y viviendas, regulando estrictamente las circunstancias en las que se pueden llevar a cabo desalojos (Comité DESC, 1997, párr. 9).

Precisamente, es el cumplimiento del conjunto de estas obligaciones derivadas de la seguridad jurídica de la tenencia lo que permite consolidar los asentamientos y el mejoramiento habitacional mediante el desarrollo de infraestructuras y servicios. En función de ello, corresponde poner de relieve la omisión de la seguridad jurídica de la tenencia - como elemento del derecho a una vivienda adecuada- en la construcción del indicador relativo a la infraestructura de la vivienda que mide el CPI, a pesar de que dicha seguridad condiciona, en los hechos, los procesos de urbanización social promovidos por el Estado.

\section{REFLEXIONES PRELIMINARES}

El desarrollo de indicadores se ha convertido en una tarea clave para dar respuesta a problemáticas que afectan la sostenibilidad y prosperidad de nuestras ciudades.

A nivel local, los indicadores se utilizan principalmente en el proceso de toma de decisiones para generar propuestas de intervención que permitan revertir dichas problemáticas. Los beneficios de la evaluación a través de indicadores de desempeño son múltiples; entre ellos, Armijo (2011) destaca los siguientes: apoya el proceso de planificación y de formulación de políticas de mediano y largo plazo; posibilita la detección de procesos o áreas en las cuales existen problemas de gestión; permite realizar ajustes en los procesos internos y readecuar cursos de acción; establece mayores niveles de transparencia respecto del uso de los recursos públicos; apoya la introducción de sistemas de reconocimiento tanto institucional como individual, entre otros beneficios.

En el marco de las definiciones en torno a una nueva agenda urbana, la utilización del Índice de la Prosperidad Urbanapromueve una serie de indicadores ligados al desarrollo urbano sostenible: productividad, infraestructura, calidad de vida, equidad e inclusión social, sustentabilidad ambiental y gobernanza y legislación urbana. Sin embargo, una de las principales limitaciones que presenta es la construcción de un subíndice de infraestructura de la vivienda que no incorpora el componente del suelo como variable central en el abordaje del problema. Esto ocurre no sólo desde la centralidad que, como hemos sostenido, tiene el acceso al suelo como eje vertebrador de la integración a la ciudad (Durand-Lasserve, 1997), sino desde la importancia que la propia Asamblea General de las Naciones Unidas ha otorgado a la incidencia del suelo urbano en el alcance de los Objetivos del Desarrollo Sostenible (principalmente al número 11: ciudades inclusivas, seguras, resilientes y sostenibles).

En definitiva, la omisión del suelo en el análisis de la dimensión relativa a la infraestructura va en detrimento del desarrollo de un enfoque holístico e integrado, esencial para la promoción del bienestar colectivo, como se expresa en diversos documentos del organismo. El análisis de esta limitación, junto con otras expuestas en el trabajo, impulsa la revisión de criterios e indicadores aplicables al contexto urbano e intraurbano.

Coincidimos en que el ejercicio realizado constituye el primer peldaño para analizar la importancia del suelo en el desarrollo de las ciudades, y que, por lo tanto, no debe omitirse. 
Sin embargo, la mera añadidura de un indicador de acceso al suelo no es suficiente para indagar sobre el problema en su complejidad, sino que es necesario avanzar hacia un abordaje integral que, a partir de un enfoque multimetódico (cuantitativo-cualitativo), incorpore efectivamente el marco conceptual de un enfoque de derechos en la producción de información para el desarrollo de políticas urbanas.

\section{REFERENCIAS BIBLIOGRÁficAS}

Abramo, P. (2003). Eu já tenho onde morar...a Cidade da informalidade. En: Abramo, P. (org.) A cidade da informalidade. O desafio das cidades latino-americanas (pp.7-23). Rio de Janeiro: Livraria Sette Letras-FAPERJ.

Abramovich, V. (2006). Una Aproximación al Enfoque de Derechos en las Estrategias y Políticas de Desarrollo de América Latina. Revista de la CEPAL, 88.

Armijo, M. (2011). Planificación Estratégica e Indicadores de Desempeño en el Sector Público. Santiago de Chile: Instituto Latinoamericano y del Caribe de Planificación Económica y Social (ILPES), CEPAL, Naciones Unidas.

Arqueros Mejica, M. S. y Canestraro, M. L. (2011). Intervenciones del Estado en la regularización de villas y asentamientos: alcances, límites y contradicciones. En Di Virgilio, M. et al. (comps.). La cuestión urbana interrogada. Transformaciones urbanas, ambientales y politicas públicas en Argentina (pp. 39-74); Buenos Aires: Editorial Café de las ciudades.

Arqueros Mejica, M.S. y Canestraro, M. L. (2017). Procesos sociales y dinámicas urbanas: un abordaje multiescalar sobre la informalidad. Revista Cardinalis, 5(9). Recuperado de https://revistas.unc.edu.ar/index.php/cardi/issue/vie $\mathrm{w} / 1555$

Brenner, N., Peck J. y Theodore, N. (2011). ¿Y después de la neoliberalización? Estrategias metodológicas para la investigación de las transformaciones regulatorias contemporáneas. Urban, 1. Recuperado de http://polired.up m.es/index.php/urban/article/view/409/1878

BID, Banco Interamericano de Desarrollo (2014). Guia metodológica; Iniciativa Ciudades Emergentes y Sostenibles (segunda edición).

Bonnefoy, J.C. y Armijo, M. (2005). Indicadores de desempeño en el sector público. Santiago de Chile: Instituto Latinoamericano y del Caribe de Planificación Económica y Social (ILPES), CEPAL, Naciones Unidas.

Buzai, G. (2003). Mapas sociales urbanos. Buenos Aires: Lugar Editorial.

Canestraro, M. L.; Zulaica, L.; Oriolani, F. y Jakubowicz, M. (2017). Intervenciones estatales y condiciones habitacionales en el Municipio de General Pueyrredon: reflexiones sobre el Distrito 5.1 Oeste Interno. Ponencia presentada al 3er Congreso Internacional de Estudios Urbanos, Argentina.

Centro de Estudios Legales y Sociales (2015). Una perspectiva de derechos sobre las políticas de desarrollo y de acceso justo al hábitat. Derechos humanos en Argentina. Informe Anual. Buenos Aires.

ONU-Hábitat (2012). State of the world's cities 2012/2013: Prosperity of cities. New York: ONU-Hábitat.

ONU- Comité de Derechos Económicos Sociales y Culturales (1991). Observación General Nro.4, El derecho a una vivienda adecuada (párrafo 1 del artículo 11 del Pacto). Recuperado de http://repositoriocdpd.net:8080/bitstre am/handle/123456789/833/Inf_NU_ObservacionDerechoViviendaAdecuada_1991.pdf?sequence=1

ONU - Comité de Derechos Económicos Sociales y Culturales (1997). Observación General Nro. 7, El derecho a una vivienda adecuada (párrafo 1 del artículo 11 del Pacto): desalojos forzosos. Recuperado de https://www.ohchr.or g/SP/Issues/Housing/Pages/ForcedEvictions.aspx

ONU- Comité de Derechos Económicos Sociales y Culturales (2002). Observación General Nro. 15, El derecho al agua (artículos 11 y 12 del Pacto Internacional de Derechos Económicos, Sociales y Culturales). Recuperado de https://www.escr-net.org/es/recursos/observacion-general-no-15-derecho-al-agua-articulos-11-y-12-del-pa cto-internacional

Dizdaroglu, D. (2015). Developing micro-level urban ecosystem indicators for sustainability assessment. Environmental Impact Assessment Review,(54). 
Donatiello, G. (2004). Environmental sustainability indicators in urban areas: An italian experience. Ottawa: National Statistical Institute of Italy.

Durand-Lasserve, A. (1997). Prólogo. En A. Azuela y T. François (Coords.), El acceso de los pobres al suelo urbano (pp. 9-12). México: CEMC-UNAM.

Fernández, R. et al. (1996). Habitar Mar del Plata. Problemática de vivienda, tierra y desarrollo urbano en Mar del Plata. Diagnóstico y propuestas. Programa Arraigo-Universidad Nacional de Mar del Plata, Comisión de Tierras Fiscales Nacionales.

Grupo de Investigación en Derechos Sociales (2017). La Corte Suprema argentina y el Pacto Informe presentado al Comité de Derechos Económicos, Sociales y Culturales. Informe presentado al Comité de Derechos Económicos, Sociales y Culturales, 61.

Hernández, D. (2017). Transporte público, bienestar y desigualdad: cobertura y capacidad de pago en la ciudad de Montevideo. Revista de la CEPAL, 122.

Holden, M. (2013). Sustainability indicator systems within urban governance: Usability analysis of sustainability indicator systems as boundary objects. Ecological Indicators, 32.

Instituto de Políticas Públicas del MERCOSUR (2014). Ganar derechos: Lineamientos para la formulación de políticas públicas basadas en derechos. Serie Documentos de Trabajo, 2.

Irigoin, M.A. (1991). La población, los habitantes y la trama social urbana, 1880-1940. En AA. VV. Mar del Plata. Una historia urbana (pp. 45-62). Buenos Aires: Fundación Banco de Boston.

Karol, J. L. (2016). El lenguaje como campo de batalla. (o ¿Qué se dice cuando se habla de 'sustentabilidad', 'equidad'o inclusión urbana'?). Actas de las IX Jornadas de Sociología de la UNLP, Argentina.

King, L. O. (2016). Functional sustainability indicators. Ecological Indicators, 66.

INDEC (2010). Censo Nacional de Población, Hogares y Viviendas. Buenos Aires: Instituto Nacional de Estadísticas y Censos

Mariano, C. (2000). Actores y prácticas económicas en las tierras rurales de Mar del Plata, 1874- 1910. Ponencia presentada al VI Congreso Argentino de Antropología Social, Mar del Plata, Argentina.

Mayer, A.L. (2008). Strengths and weaknesses of common sustainability indices for multidimensional systems. Environ. Int., 34(1).

Merlisnky, G. (2013). Politica, derechos y justicia ambiental. El conflicto del Riachuelo. Buenos Aires: Fondo de Cultura Económica.

Mori, K. and Christodoulou, A. (2012). Review of sustainability indices and indicators: Towards a new City Sustainability Index (CSI). Environmental Impact Assessment Review, 32.

Núñez, A. (1994). Implicancias sociales de los procesos de apropiación de la tierra en Mar del Plata (Informe Final Beca de Perfeccionamiento). Universidad Nacional de Mar del Plata.

Núñez, A. (2000). Morfología social. Mar del Plata, 1874-1990. Tandil: Grafikart.

ONU-Hábitat (2012). State of the world's cities 2012/2013: Prosperity of cities. New York: ONU-Hábitat.

ONU-Hábitat (2016a). Índice de Prosperidad Urbana en la República Mexicana. México: ONU-Hábitat.

ONU-Hábitat (2016b). La prosperidad en las ciudades de Ecuador: Primer reporte del Índice de Prosperidad Urbana (CPI) para 27 ciudades ecuatorianas. Quito: ONU-Hábitat - Banco de Desarrollo de América Latina.

Pautassi, L. (2016). Indicadores de progreso para medir derechos sociales: un nuevo escenario para las políticas públicas en América Latina. En Muñoz-Pogossian B. y Barrantes A. (eds.). Equidad e Inclusión Social: superando desigualdades hacia sociedades más inclusivas (pp. 35 - 57). Washington: Secretaría General de la Organización de los Estados Americanos.

Pautassi, L. (2013); Monitoreo del acceso a la información desde los indicadores de derechos humanos. Sur. Revista Internacional de Derechos Humanos, 18.

Pírez, P. y Salazar, C. (2016). Para entender la urbanización en América Latina: el predominio de la mercantilización del suelo urbano. Ponencia presentada en III Seminario Internacional de la Red Latinoamericana de Investigadores sobre Teoría Urbana, México. 
Reyers, B., Stafford-Smith, M., Heinz Erb, K., et al. (2017). Essential Variables help to focus Sustainable Development Goals monitoring. Current Opinion in Environmental Sustainability.

Rodríguez, A. y Sugranyes, A. (2017). La Nueva Agenda Urbana, pensamiento mágico. Revista Vivienda y Ciudad, 4. Recuperado de https://revistas.unc.edu.ar/index.php/ReViyCi/article/view/19005

Rozas Balbontín, P. y Salazar Arredondo, L. (2015). Violencia de Género en el Transporte Público: una regulación pendiente. CEPAL - Serie Recursos Naturales e Infraestructura, (172). Recuperado de https://ppp.worldbank.org/public-private-partnership/sites/ppp.worldbank.org/files/documents/Violenci a\%20de\%20ge\%CC\%81nero\%20en\%20el\%20transporte\%20pu\%CC\%81blico_ES.pdf

Schuschny, A. y Soto, H. (2009). Guía metodológica, diseño de indicadores compuestos de desarrollo sostenible. Santiago de Chile: CEPAL, Naciones Unidas.

Shen, L.Y., Ochoa, J.J., Shah, M.N. and Zhang, X. (2011). The application of urban sustainability indicators-a comparison between various practices. Habitat International, 35.

Spangenberg, J. y Bonniot, O. (1998). Sustainability Indicators: A compas son the road towards sustainability. Wuppertal: Wuppertal Institute.

Srebotnjak, T.; Polzin, C.; Giljum, S.; Herbert, S. y Lutter, S. (2010). Establishing Environmental Sustainability Thresholds and Indicators. Final report. Ecologic Institute and SERI.

Trivelli, P. (2007). Monitoreo del suelo urbano y de los mercados de construcción. En Smolka M. y Mullahy L. (eds.). Perspectivas urbanas. Temas criticos en políticas de suelo en América Latina (pp. 29-30). CambridgeMassachusetts: Lincoln Institute of Land Policy.

Vázquez, D. y Delaplace, D. (2011). Políticas públicas con perspectiva de derechos humanos. Un campo en construcción. Revista SUR, 14.

Wood, S.L.R., Jones, S.K., Johnson, J.A. et al. (2018). Distilling the role of ecosystem services in the Sustainable Development Goals. Ecosystem Services, 29.

\section{Notas}

1 Cabe mencionar que una versión preliminar de este artículo fue discutida en el 3er Congreso Internacional de Vivienda y Ciudad: Debate en torno a la nueva agenda urbana, organizado por el INVIHAB (Instituto de Investigaciones de Vivienda y Hábitat) y la Maestría en Gestión y Desarrollo Habitacional/Universidad Nacional de Córdoba. Realizado en Córdoba, 21 y 22 de junio de 2018.

2 Hasta el momento se ha puesto en funcionamiento el monitoreo de desempeño de políticas en seguridad social, salud, educación, etc. (Pautassi, 2016), pero no existen antecedentes para el caso de las políticas habitacionales. Sí encontramos como experiencia sumamente relevante la de la Autoridad de la Cuenca Matanza - Riachuelo (ACUMAR), originada en 2004 a partir de una demanda presentada por vecinos de Villa Inflamable y trabajadores de la salud del Municipio de Avellaneda (Buenos Aires, Argentina) que puso en escena el carácter colectivo del derecho al ambiente sano (Merlinsky, 2013).

3 Ambas autoras destacan además la connivencia con el poder político provincial, que no sólo avaló la transgresión de la normativa para fundar el pueblo - que no se hizo sobre tierras públicas, tal como lo establecía la Ley de Ejidos-, sino que prosiguió con esta impronta en otras intervenciones relativas a la movilización de tierras (Mariano, 2000; Núñez, 2000).

4 Con la constitución del Foro Comunitario de Seguridad en el ámbito del Partido de General Pueyrredón, en 1997, mediante el decreto 1979 se define la división del mismo en distritos, acorde a las zonas de influencia o circuitos de las comisarías. Posteriormente, a partir de 2002, al comenzar a debatirse sobre la necesidad de contar con un Presupuesto Participativo, se tiene en consideración aquella división. Actualmente, el PGP se encuentra dividido en 16 distritos: 1.6 Centro, 2 Norte, 3 Centro Oeste, 4 Noroeste, 5.1 Oeste Interno, 5.2 Oeste Externo 7.1 Suroeste Interno, 7.2 Suroeste Externo, 8.1 Puerto Interno, 8.2 Puerto Externo, 9 Batán, Ruta 11 Sur A/M, Ruta 11 Sur Sur, Ruta 11 Norte, Ruta 2 y Ruta 226. El Oeste Interno comprende los barrios Caribe, Dorrego, Las Américas, Las Lilas, Newbery, Regional, Belisario Roldan, San Cayetano y Santa Rosa De Lima

5 Se elaboraron mapas y gráficos que permiten representar la incidencia del indicador referido a la propiedad del terreno y la vivienda en la subdimensión infraestructura de vivienda, en el marco de la metodología de ciudades prósperas. Las categorías empleadas en los mapas se definieron mediante la clasificación por cortes naturales. Este método identifica los puntos de ruptura 
entre las clases utilizando una fórmula estadística (optimización de Jenk), que minimiza la suma de la varianza dentro de cada una de las clases.

6 De hecho, al correlacionar el indicador propiedad del terreno y la vivienda con los restantes, se observan valores bajos y próximos a cero del coeficiente de correlación lineal ( $r$ de Pearson), lo cual pone en evidencia una distribución aleatoria. Esto significa que la distribución de este indicador no presenta semejanzas con los restantes. Asimismo, cuando se obtiene el promedio estandarizado de los cuatro indicadores contenidos en el modelo y el correspondiente a la propiedad del terreno y la vivienda, se presenta el valor más bajo en este último.

7 Abramo (2003) distingue tres lógicas en relación al acceso al suelo: de Estado, por la cual los individuos se someten a una decisión del poder público que asume la responsabilidad de un mejor grado de bienestar social; de mercado, en el que se encontrarían quienes desean consumir tierra urbana y quienes disponen de tierra y la ofrecen a los eventuales consumidores, $y$ de la necesidad, esto es, los procesos de acción colectiva de ocupaciones de terrenos e inmuebles que se originan por la incapacidad de acceder a través del mercado (formal o informal) y por la ausencia de respuesta por parte del Estado.

8 Por ejemplo, tal como ha estudiado Azuela (1999) en relación a aquellas ocupaciones de tierra -como parte de la lógica de la necesidad- que luego devienen en un proceso de configuración de un mercado, o, como dice Cravino (2006), de "mercantilización de las relaciones de reciprocidad".Ello pone en evidencia las superposiciones y reconversiones.

9 El art. 25.1 de la Declaración Universal de Derechos Humanos, afirma que: "Toda persona tiene derecho a un nivel de vida adecuado que le asegure, así como a su familia, la salud y el bienestar, y en especial la alimentación, el vestido, la vivienda, la asistencia médica y los servicios sociales necesarios; tiene asimismo derecho a los seguros en caso de desempleo, enfermedad, invalidez, viudez, vejez u otros casos de pérdida de sus medios de subsistencia por circunstancias independientes de su voluntad". Por su parte, el art. 11, primer párrafo del Pacto Internacional de Derechos Económicos, Sociales y Culturales, establece que: "Los Estados Parte en el presente Pacto reconocen el derecho de toda persona a un nivel adecuado de vida para sí y su familia, incluso alimentación, vestido y vivienda adecuados, y a una mejora continua de las condiciones de existencia. Los Estados Partes tomarán medidas apropiadas para asegurar la efectividad de este derecho, reconociendo a este efecto la importancia esencial de la cooperación internacional fundada en el libre consentimiento".

10 Los principios de igualdad y no discriminación están consagrados en los artículos $1^{\circ}$ y $2^{\circ}$ de la Declaración Universal de Derechos humanos; en los artículos $1^{\circ}, 2^{\circ}$ y 26 del Pacto Internacional de Derechos Civiles y Políticos; en los artículos $1^{\circ}$ y $2^{\circ}$ del Pacto Internacional de Derechos Económicos, Sociales y Culturales; en el artículo II de la Declaración Americana de los Derechos y Deberes del Hombre y en los artículos $1^{\circ}$ y 24 de la Convención Americana sobre Derechos Humanos. Dos convenciones internacionales los consagran de forma más específica: la Convención Internacional sobre la Eliminación de todas las Formas de Discriminación Racial y la Convención sobre la Eliminación de todas las Formas de Discriminación contra la Mujer.

11 El principio de participación surge del artículo 21 de la Declaración Universal de Derechos Humanos, el artículo 25 del Pacto Internacional de Derechos Civiles y Políticos y el artículo 13.1 del Pacto Internacional de Derechos Económicos, Sociales y Culturales. Asimismo, diversos instrumentos internacionales sobre pueblos indígenas consagran de manera específica su derecho a ser consultados: por ejemplo, los artículos 2, 5, 6 y 7 del Convenio núm. 169 sobre pueblos indígenas y tribales en países independientes de la Organización Internacional del Trabajo (OIT, 1989) y los artículos 5, 18, 23, 27 y 43 de la Declaración de las Naciones Unidas sobre los derechos de los pueblos indígenas. 